\title{
Abstract: HoloLens \\ Streaming of 3D Data from Ultrasound Systems to Augmented Reality Glasses
}

\author{
Felix von Haxthausen ${ }^{1}$, Floris Ernst ${ }^{1}$, Ralf Bruder ${ }^{1}$, Mark Kaschwich ${ }^{2}$ \\ Verónica García-Vázquez ${ }^{1}$ \\ ${ }^{1}$ Institut für Robotik und Kognitive Systeme, Universität zu Lübeck \\ ${ }^{2}$ Division of Vascular- and Endovascular Surgery, Department of Surgery, \\ University Hospital Schleswig-Holstein, Campus Lübeck \\ vonhaxthausen@rob.uni-luebeck.de
}

Two-dimensional ultrasound (US) imaging is one of the most common tools for diagnostic procedures. However, this imaging modality requires highly experienced and skilled operators to mentally reconstruct three-dimensional (3D) anatomy from these images. Additionally, the physician's gaze is focused on the screen of the US system instead of the probe and patient. In order to overcome these problems, we propose real-time 3D US in combination with augmented reality (AR) glasses (specifically Microsoft HoloLens) to render the volume relative to the US probe [1]. Raw data access to the US system was provided by an in-house modification and volumes were sent from the US system (GE Vivid 7) to a computer via Ethernet. On the computer, the data was first converted from spherical to Cartesian coordinates and then the zeros (mainly presented outside the pyramid-shaped US volume) were compressed using run-length encoding. A multi-language remote procedure call (gRPC) was used to transmit the encoded data from the computer to HoloLens via Wi-Fi. The AR marker attached to the US probe, which allows the placement of the virtual volume next to the probe, was tracked using HoloLensARTToolkit. This approach was evaluated regarding the rate of displayed volumes on HoloLens and the end-to-end latency. US volumes (depth of $15 \mathrm{~cm}$, matrix size in spherical coordinates of $495 \times 72 \times 26$ ) were acquired at a frame rate of $13.8 \mathrm{~Hz}$. On HoloLens, the volumes had a matrix size in Cartesian coordinates of $103 \times 74 \times 134$. Our results show that the volumetric data was rendered with a time interval of $72 \pm 55 \mathrm{~ms}$ and an end-to-end latency of $259 \pm 85 \mathrm{~ms}$. The loss-less data encoding allowed a decrease of $63 \%$ in data size. There is a growing interest in using real-time 3D US for clinical applications and HoloLens gives the possibility of a more intuitive visualization of volumetric data than using a standard screen. Future work will evaluate the AR marker tracking and other transmissions paths in order to lower the end-to-end latency.

\section{References}

1. von Haxthausen F, Ernst F, Bruder R, et al. Streaming of 3D data from ultrasound systems to augmented reality glasses (HoloLens). Proc CURAC. 2018; p. 2-3. 